\title{
Word cues affect detection but not localization responses
}

\author{
KAREN MORTIER \\ Vanderbilt University, Nashville, Tennessee
}

AND

Wieske van Zoest, MartiJn Meeter, AND Jan TheEUWeS
$V U$ University, Amsterdam, The Netherlands

\begin{abstract}
Many theories assume that preknowledge of an upcoming target helps visual selection. In those theories, a top-down set can alter the salience of the target, such that attention can be deployed to the target more efficiently and responses are faster. Evidence for this account stems from visual search studies in which the identity of the upcoming target is cued in advance. In five experiments, we show that top-down knowledge affects the speed with which a singleton target can be detected but not the speed with which it can be localized. Furthermore, we show that these results are independent of the mode of responding (manual or saccadic) and are not due to a ceiling effect. Our results suggest that in singleton search, top-down information does not affect visual selection but most likely does affect response selection. We argue that such an effect is found only when information from different dimensions needs to be integrated to generate a response and that this is the case in singleton detection tasks but not in other singleton search tasks.
\end{abstract}

In everyday life, we are constantly subjected to massive visual stimulation from the environment. In order to behave in a goal-directed manner, it is important to select only relevant information from the environment, to ignore information that is irrelevant. Selection of visual information that accords with our goals is referred to as top-down control of selection. However, irrelevant but salient items can also capture our attention, such as when one sees movement in the corner of the eye and feels compelled to look at what is moving. This is called bottom-up control of selection (for reviews, see, e.g., Corbetta \& Shulman, 2002; Rauschenberger, 2003; Theeuwes \& Godijn, 2001).

Although there is little controversy regarding the importance of bottom-up factors such as local feature contrast in selection, there is much less consensus about how these bottom-up factors interact with top-down modulation. More precisely, it is hotly debated whether top-down control modulates the selection of salient stimuli immediately or whether top-down control affects performance only after a salient stimulus has been selected on the basis of bottom-up control.

Evidence for the former stems from studies in which the amount of information available about the target identity in singleton search tasks was manipulated (Egeth, 1977; Meeter \& Theeuwes, 2006; Müller, Heller, \& Ziegler, 1995; Müller, Reimann, \& Krummenacher, 2003; Theeuwes \& Van der Burg, 2007; Treisman, 1988). A typical finding is that search is faster when observers know the identity of the target dimension within a block of trials than when the target dimension changes from trial to trial; this is referred to as a cross-dimensional cost. Although many have argued that cross-dimensional cost may result from bottom-up priming rather than from top-down modulation (e.g., Müller et al., 2003; Theeuwes, Reimann, \& Mortier, 2006), Müller et al. (2003) presented evidence that top-down knowledge does affect performance, even when bottom-up priming cannot play a crucial role. In their study, the target dimension was cued with a picture cue before each trial. When the cue indicated the upcoming target dimension, performance was better than when the cue was invalid. These results, since replicated by others (Meeter \& Theeuwes, 2006; Müller \& Krummenacher, 2006; Theeuwes et al., 2006, Experiment 1), were taken as strong support for top-down control in visual search (Müller et al., 2003).

However, other studies suggest that these effects are not the result of top-down guidance of attention but represent effects occurring later in time (i.e., after the target has been selected for further processing; e.g., Cohen \& Magen, 1999; Mortier, Theeuwes, \& Starreveld, 2005; Theeuwes et al., 2006). In these studies, the response requirements were manipulated. For example, Theeuwes et al. (2006) employed the standard visual search singleton detection task (i.e., was the target singleton present or not?) but also employed a compound search task in which participants 
had to discriminate the orientation of a line segment that was presented inside the target singleton. A compound task disentangles the visual selection processes from the response selection processes, since the target-defining search attribute is not the same as the response-defining attribute (cf. Duncan, 1985). If knowledge of the target dimension affects visual selection, a cuing effect should have been observed in compound search tasks, as well as in simple detection tasks. However, there was a clear cuing effect only in the detection task, whereas in the compound task, this cuing effect was absent (Theeuwes et al., 2006). In a similar vein, Kumada (2001, Experiments 1A and 1B) showed cross-dimensional costs in a simple detection task but not in a compound search task. These results suggest that early visual selection was not modulated by top-down control (see also Theeuwes \& Van der Burg, 2007). Previously reported cuing effects (e.g., Müller et al., 2003) may reflect effects on processes occurring after the selection of the target had already occurred (Mortier et al., 2005).

Nevertheless, the extent to which top-down knowledge affects performance in compound search tasks is still a matter of debate. Müller and Krummenacher (2006) recently reported data showing a small effect of top-down knowledge in a compound search task. They suggested that others failed to find such an effect because compound tasks are more difficult to perform than detection tasks and because it may be hard to observe the effects of top-down modulation in difficult tasks. The argument is that in complex tasks, such as in compound search tasks, top-down effects remain hidden. In line with this conjecture, reaction times (RTs) are usually slower in compound search tasks (between 500 and $700 \mathrm{msec}$ ) than in detection tasks (between 400 and $500 \mathrm{msec}$ ).

In the present study, we addressed whether task complexity does play a role in obtaining top-down effects in singleton search, as was assumed by Müller and Krummenacher (2006). If increasing the complexity of the task - for example, by changing a simple task into a compound search task (Mortier et al., 2005; Theeuwes et al., 2006) - hides top-down effects, one would expect that top-down effects should be observed in all simple and fast search tasks. In the present study, we determined the top-down effects for two very simple tasks: a classic target detection task and a target localization task. The advantage of a localization task is that it is the simplest form of compound search that participants can perform at high speeds. This is particularly true when the required response is an eye movement to the target. Saccadic RTs in such search tasks can be below $200 \mathrm{msec}$. If word cues affect the speed with which targets can be selected and if this effect is only observed with simple, fast tasks, one should expect to find an effect of cue validity when participants simply make a saccade to cued targets. In other words, if preknowledge about the upcoming target boosts the salience signal of the target, as is assumed by the dimensional weighting account (Krummenacher, Müller, \& Heller, 2002; Müller et al., 1995; Müller et al., 2003) or by theories that assume top-down prioritizing (e.g., Wolfe, Butcher, Lee, \& Hyle, 2003), one would expect that the saccade latency to the target would be reduced when the cue provided valid information about the properties of the upcoming target.

In all of the experiments in the present study, we made use of a feature singleton search. In such tasks, the target has a unique basic feature (e.g., a tilted line surrounded by vertical line elements) and therefore pops out from the display. The question is whether top-down information modulates the salience signal of the pop-out target. In all but Experiment 3, a word cue (as was used by Müller et al., 2003) indicated the dimension of the following target. We replicated the results of Müller et al. (2003) using a manual singleton detection task (Experiment 1A) and a saccadic singleton detection task (Experiment 1B). In Experiment 2, participants made a speeded saccade to the location of the singleton. If top-down knowledge affects the speed with which attention can be directed to the target location (as is assumed by top-down theories of visual search), one would expect an effect of advance knowledge on saccade-to-target latencies. Experiments 3 and 4 were conducted to address possible ceiling effects in the saccade-to-target latencies. Experiment 3 was identical to Experiment 2, except that a picture cue was used instead of a word cue. In Experiment 4, participants had to localize the singleton target with a manual response.

\section{GENERAL METHOD}

\section{Participants}

In total, 52 participants, ranging in age from 18 to 38 years, participated as paid volunteers. All of the participants had normal or corrected-to-normal vision and were naive to the purpose of the experiment. The numbers of participants tested in each experiment were 10 for Experiments $1 \mathrm{~A}$ and 2-4, and 12 for Experiment 1B.

\section{Apparatus}

The participants were seated in front of a computer monitor with their heads fixed on a chinrest. Viewing distance was approximately $75 \mathrm{~cm}$. The participants' eye movements were recorded using an Intelligent Eye Tracking System (SR Research, Eyelink I, 250-Hz temporal resolution and $0.2^{\circ}$ spatial resolution). Saccades were identified by means of a velocity threshold ( $35 \mathrm{deg} / \mathrm{sec})$ and an acceleration threshold $\left(9,500 \mathrm{deg} / \mathrm{sec}^{2}\right)$. The eyetracking system was calibrated at the start of the experiment and following each break. The participants were instructed not to move their eyes during the trials. Trials in which an eye movement of $3^{\circ}$ or more was made were removed from the data analysis. Between the trials, the participants were allowed to move their eyes.

\section{Stimuli}

The stimuli were presented on a black background. White vertical line segments (nontargets) were arranged in a $9 \times 13$ rectangular matrix $\left(15.9^{\circ} \times 13.2^{\circ}\right)$. The line segments had an approximate height of $0.76^{\circ}$ and an approximate width of $0.15^{\circ}$. The target was either a green vertical line segment (color target; CIE $x$ - and $y$-coordinates of 0.300 and $0.599 ; 14.85 \mathrm{~cd} / \mathrm{m}^{2}$ ) or a $45^{\circ}$ right-tilted white line segment (orientation target; CIE $x$ - and $y$-coordinates of 0.288 and 0.316 ; $93.14 \mathrm{~cd} / \mathrm{m}^{2}$ ) and could appear at six different fixed locations. These six potential locations (three on the left of the vertical midline and three on the right) were equidistantly placed on an imaginary circle in such a way that, embedded in the matrix of nontargets, the target was always presented at equal eccentricity from fixation $\left(4.3^{\circ}\right)$.

\section{Procedure}

In order to start each trial, the participants had to maintain fixation on a central point and press the space bar. Once the space bar 


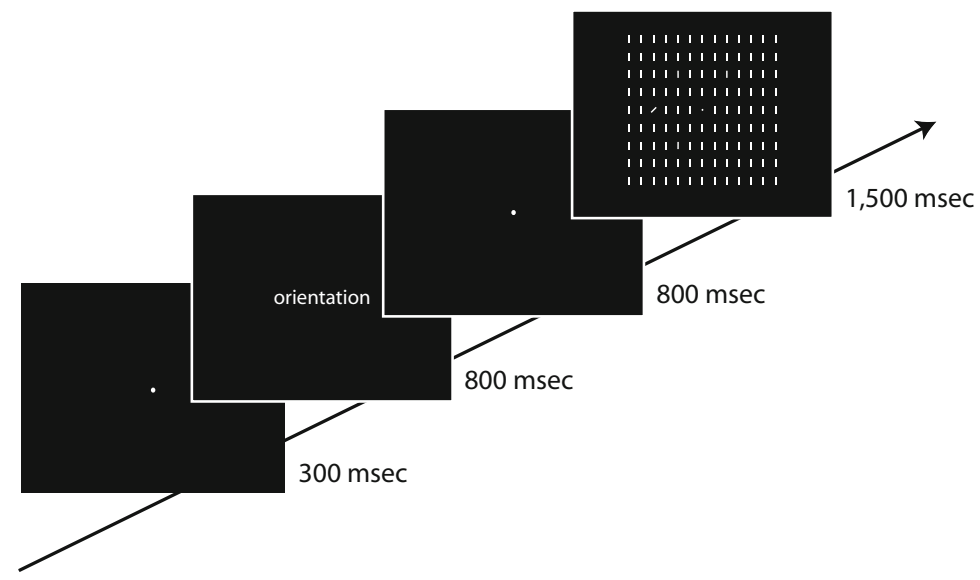

Figure 1. An example of a trial sequence of Experiment 1A. A fixation dot was presented for $300 \mathrm{msec}$, followed by the cue display (COLOR, ORIENTATION, or NEUTRAL) presented for 800 msec on a black background. The fixation point was presented again for $800 \mathrm{msec}$, followed by the stimulus display for $1,500 \mathrm{msec}$.

was pressed, the trial began with the presentation of a fixation point for $300 \mathrm{msec}$. A cue was presented for $800 \mathrm{msec}$ in the center of the visual display, followed by the fixation point for $800 \mathrm{msec}$. The stimulus display was presented for $1,500 \mathrm{msec}$. It was emphasized that they should use the cue in order to reduce RTs. Figure 1 depicts the sequence of the events of a trial.

\section{Design}

The participants started the experiment with 48 practice trials. All of the observers participated in the valid cue and the neutral cue conditions. In all experiments, except for Experiment 3, the cue in the valid cue condition was either COLOR or ORIENTATION. In the neutral cue condition, the cue was the word NEUTRAL. In Experiment 3 , a picture cue was used. The picture cue in the valid condition was identical to the target singleton. In the neutral condition, a large white dot was presented as an uninformative cue.

These two conditions were blocked and presented in a counterbalanced order across participants. Each cue condition comprised 10 experimental blocks. Each block consisted of 30 trials in Experiments 1A, 2, and 3 (total: 600 trials), 24 trials in Experiment 1B (total: 480 trials), and 21 trials in Experiment 4 (total: 420 trials). Color-target trials $(25 \%)$, orientation-target trials (25\%), and targetabsent trials $(50 \%)$ were randomly intermixed in all blocks of Experiments $1 \mathrm{~A}$ and $1 \mathrm{~B}$. In Experiments 2-4, the target was always present, with color targets and orientation targets presented equally often. At the end of each block, there was a short break, during which the participants received feedback on their accuracy and RTs.

\section{EXPERIMENTS 1A AND 1B}

Our aim in Experiments 1A and 1B was to investigate whether it is possible to obtain the typical cuing effect with the present stimuli and a word cue. The observers had to discern the presence or absence of a singleton target. We were especially interested in whether we would find the same cuing effect with saccadic responses as with manual responses. In Experiment 1A, the participants responded with a manual keypress, whereas in Experiment 1B, they responded by moving their eyes either up or down.

In each trial, a word cue either indicated with $100 \%$ validity the dimension of the upcoming target or was unin- formative regarding the target dimension. Note that unlike earlier researchers, we used a $100 \%$ valid cue (i.e., there were no invalid cues), which was compared with a neutral cue. The participants thus had every reason to use this cue to speed up their response.

\section{Method}

In Experiment 1A, the observers were told to respond to the presence or absence of the target as quickly as possible with a left manual response ("z" button) or a right manual response ("/" button). The observers were told not to make eye movements during a trial. In Experiment 1B, the participants had to indicate the presence or absence of the target by moving their eyes either up or down. For half of the participants, an upward eye movement was required when a target was present, whereas a downward movement was required when no target was presented. The other half of the participants were required to make an eye movement down when the target was present and up when the target was absent. The response area was defined by two circles with a radius of $3^{\circ}$ positioned at an eccentricity of $7^{\circ}$ on the vertical axis at the top and bottom of the display (see Figure 2). When the participants' eyes entered the response interest area, a click sound indicated to the participants that they had successfully triggered a response. The participants were told to ignore the singleton targets and were encouraged to respond as accurately and quickly as possible.

\section{Results}

Experiment 1A. RTs longer than 1,000 msec (0.24\%) and trials in which an eye movement was made (5.38\%) were excluded from the analysis. Trials with incorrect responses $(3.46 \%)$ were submitted to an ANOVA, with the factors cue (valid or neutral), target presence (present or absent), and target dimension (orientation or color). There were no effects of cue or target dimension $\left(F_{\mathrm{S}}<1\right)$. There was a main effect of target presence $[F(1,9)=11.82, p<$ $.01]$. There were more misses $(4.2 \%)$ than false alarms $(2.7 \%)$.

An ANOVA was performed on the individual mean RTs of target-present trials with cue and target dimension as factors. There were main effects of cue $[F(1,9)=$ 


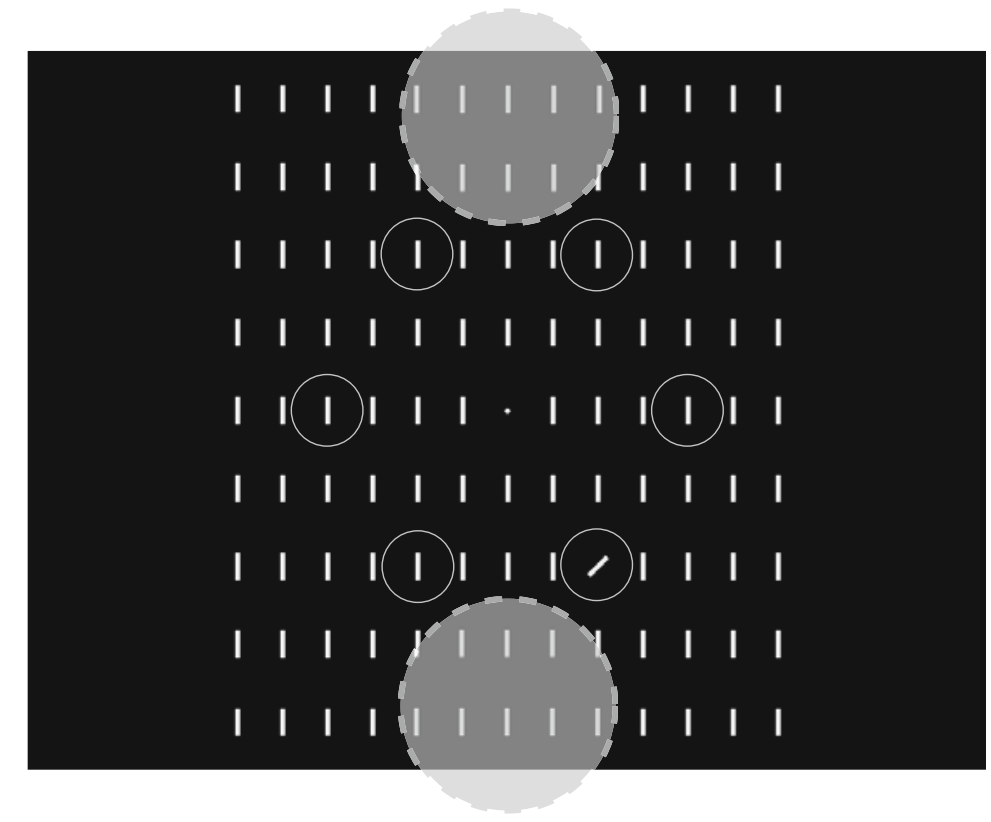

Figure 2. Example of the display used in Experiment 1B. The possible target locations are indicated by small solid circles. The two response areas in which the eyes had to land are indicated by dashed circles at the top and bottom.

$10.66, p=.01]$ and of target dimension $[F(1,9)=9.16$, $p<.05]$. Responses were faster in the valid cue condition $(M=402 \mathrm{msec})$ than in the neutral cue condition $(M=$ $422 \mathrm{msec})$. Color targets $(M=404 \mathrm{msec})$ were responded to faster than orientation targets $(M=420 \mathrm{msec})$. There was an interaction between cue and target dimension $[F(1,9)=5.71, p<.05]$, with larger cuing effects for color targets than for orientation targets. (See Table 1.)

RTs on the target-present trials $(M=412 \mathrm{msec})$ were not shorter than RTs on the target-absent trials $(M=$ $423 \mathrm{msec})(F<1)$, providing evidence that the present task was a singleton detection task (see Theeuwes, Kramer, \& Atchley, 1999). An ANOVA on the RTs of the target-absent trials with cue as a factor revealed no effect $[F(1,9)=1.39$, n.s. $]$.

Experiment 1B. Saccadic latencies below $80 \mathrm{msec}$ (anticipation errors, 5.45\%) and saccadic latencies above $600 \mathrm{msec}(1.20 \%)$ were excluded from the analysis. Initial saccades with an endpoint located within $1^{\circ}$ of a target position were regarded as captured eye responses and were not analyzed further $(1.37 \%){ }^{1}$

Incorrect eye responses were made in $6.35 \%$ of all trials. For example, the participants made an eye movement down when the target was present but had been instructed to make an eye movement up when the target was present. An ANOVA was performed on the incorrect eye responses in Experiment 1B, with the factors cue (valid or neutral) and target dimension (color or orientation). There were no significant differences: No effect of cue $[F(1,11)=2.56, p=.14]$, no effect of target dimension $[F(1,11)=1.82, p=.2]$, and no interaction effect $(F<1)$ was found.
A within-subjects ANOVA was performed on the saccadic latencies, with cue (valid or cue neutral) and target dimension (color or orientation) as factors. The main effect of target dimension was significant $[F(1,11)=8.59$, $p<.05]$, with faster latencies for color targets $(M=$ $317 \mathrm{msec})$ than for orientation targets $(M=332 \mathrm{msec})$. There was a significant main effect of cue $[F(1,11)=$ $7.06, p<.05]$. Furthermore, there was an interaction between cue and target dimension $[F(1,11)=9.94, p<.01]$. The obtained cuing effect was stronger for color targets than for orientation targets. (See Table 1.)

\section{Discussion}

In Experiment 1A, in which we used a classic target detection task, the conventional benefit of cuing the upcoming target was obtained: The observers were faster to respond manually when the cue indicated the dimension of the target than when the cue was uninformative. Experiment $1 \mathrm{~B}$ shows that preknowledge about the target dimension also facilitates saccadic detection performance. As in Experiment 1A, a valid cue speeded up detection performance relative to a neutral cue. Therefore, the word cue was effective in inducing a top-down setting regarding the dimension of the target, independent of the mode of responding.

Also, in Experiments 1A and 1B, a color target was detected faster than an orientation target. This result is in agreement with previous results (Found \& Müller, 1996; Hillstrom, 2000; Mortier et al., 2005; Theeuwes et al., 2006) and is probably due to the fact that a color target is more salient than an orientation target (see also Olivers \& Humphreys, 2003; Theeuwes, 1992). Furthermore, the 
Table 1

Mean Reaction Time (in Milliseconds) for Target-Present Trials As a Function of the Word Cue (Present or Absent) and Target Dimension (Color or Orientation)

\begin{tabular}{lcc}
\hline & \multicolumn{2}{c}{ Word Cue } \\
\cline { 2 - 3 } Target Dimension & Valid & Neutral \\
\hline \multicolumn{2}{c}{ Experiment 1A: Manual Response } \\
Color & $389^{*}$ & $419^{*}$ \\
Orientation & 415 & 424 \\
Experiment 1B: Eye Movement & \\
Color & $306^{*}$ & $329^{*}$ \\
Orientation & 328 & 337 \\
\multicolumn{2}{c}{ Experiment 2: Eye Movement } \\
Color & 180 & 185 \\
Orientation & 192 & 195 \\
Experiment 4: Manual Response & \\
Color & 411 & 410 \\
Orientation & 414 & 409
\end{tabular}

${ }^{*} p \leq .05$.

color targets differed from the distractors in hue, as well as in luminance, which might have created a redundancy gain (e.g., Krummenacher, Müller, \& Heller, 2001). The obtained cuing effect was stronger for color targets than for orientation targets.

The present results replicate the findings of Müller et al. (2003; Theeuwes et al., 2006, Experiment 1) and are consistent with theories that assume that top-down knowledge can improve visual search for a singleton target (Found \& Müller, 1996; Müller et al., 1995; Müller et al., 2003; Wolfe et al., 2003). More specifically, because processing is tuned to a specific dimension (i.e., the cued dimension), it is assumed that attention can be deployed faster to the target singleton.

\section{EXPERIMENT 2}

If cuing speeds up the covert deployment of attention to singleton targets (e.g., Müller et al., 2003; Wolfe et al., 2003; Wolfe, Horowitz, Kenner, Hyle, \& Vasan, 2004), one should also see this effect in overt attentional deployment. Indeed, one would expect that preknowledge of the target properties would boost the salience signal, allowing a faster deployment of the saccade, generating faster eye latencies. If top-down knowledge of the target identity speeds up the allocation of attention, saccadic latencies should decrease when the identity of the target is cued.

\section{Method}

The same cues and stimuli as those in Experiments 1A and 1B were used, but now, the observers were instructed to make a speeded eye movement to the singleton target. As soon as the stimulus display was presented, the participants had to make a speeded eye movement as quickly and as accurately as possible to the target.

\section{Results}

Of the saccades, $1.85 \%$ missed the target location (outside $3^{\circ}$ of visual angle of the target position). Those saccades were not analyzed further. Saccadic latencies below $80 \mathrm{msec}$ (anticipation errors, 6.96\%) or above $600 \mathrm{msec}$
$(0.29 \%)$ were counted as errors and were excluded from the saccadic latencies analysis.

A within-subjects ANOVA was performed on the correct saccadic latencies, with cue (valid or neutral) and target dimension (color or orientation) as factors. The main effect of target dimension was significant $[F(1,9)=$ 46.44, $p<.001$ ], with faster latencies for color targets $(M=182.5 \mathrm{msec})$ than for orientation targets $(M=$ $193.5 \mathrm{msec})$. There was no main effect of cue $[F(1,9)=$ $1.06, p=.33]$. There was no interaction between cue and target dimension $[F(1,9)<1]$. (See Table 1.)

The anticipation errors (saccadic latencies below $80 \mathrm{msec}$; 6.69\%) were submitted to an ANOVA with cue (valid or neutral) and target dimension (color or orientation) as factors. There was a main effect only of cue $[F(1,9)=5.92, p<.05]$, with more errors if the cue was valid $(M=8.7 \%)$ than if the cue was neutral $(M=5.3 \%)$. This result suggests that the participants tried to actively use the cue in order to optimize their performance. Note, however, that almost none of these ultrafast eye movements landed at the target location (0.3\%), implying that the cue did not benefit these rapid responses in any way. There was no effect of target dimension $(F<1)$. The interaction between the two factors was not significant $(F<1)$.

\section{Discussion}

If advance top-down knowledge modulates the salience of the signals in the visual field (e.g., Müller et al., 2003; Wolfe et al., 2003; Wolfe et al., 2004), knowledge of the upcoming target should decrease saccadic latencies of eye movements directed to the cued target. However, the results of Experiment 2 clearly show that preknowledge about the dimension of the upcoming target did not affect saccadic latency (i.e., $F \mathrm{~S} \approx 1$ ). Note that the very same cues were effective in Experiment 1, in which a detection response was required. The data suggest that word cues that have an effect on singleton detection task (Experiment 1) do not affect a localization task, as was used in Experiment 2.

\section{EXPERIMENT 3}

One may argue that Experiment 2 cannot be compared with Experiment 1B, because they differ in RTs. Saccadic latencies in Experiment 2 were around $200 \mathrm{msec}$, whereas the saccadic detection RTs in Experiment 1B were around $325 \mathrm{msec}$. It is possible that top-down information could not affect saccade-to-target latencies for the simple reason that there was no room for improvement: The saccades could not be executed any faster. In other words, it is possible that no top-down cuing effects were observed because of possible ceiling effects.

There is evidence that saccades to a pop-out target are quicker and more accurate when the target has the same color as on previous trials than when the target color switches (McPeek, Maljkovic, \& Nakayama, 1999; see also Bichot \& Schall, 2002). Thus, it should be possible to modulate saccadic latencies in a saccadic localization 
task by cuing the target with the actual target singleton. Experiment 3 was conducted to investigate this issue. This experiment was identical to Experiment 2, except that the word cue was replaced by the actual target singleton.

\section{Method}

The stimuli, design, and procedure were the same as those in Experiment 2 , with only one change. Instead of a word cue, a picture cue was used. The picture cue in the valid condition was identical to the target singleton and was presented in the center of the screen. In the neutral condition, a large white dot was presented as an uninformative cue.

\section{Results}

Saccadic latencies below $80 \mathrm{msec}$ (anticipation errors, $5.65 \%)$ and saccadic latencies above $600 \mathrm{msec}(0.66 \%)$ were counted as errors and were excluded from the analysis. There were $6.33 \%$ of the saccades that missed the target location (outside $3^{\circ}$ of visual angle of the target position). Those saccades were not analyzed further.

An ANOVA was performed on the correct saccadic latencies with target dimension (color or orientation) and cue (valid or neutral) as factors. The main effect of target dimension was significant $[F(1,9)=11.7, p<.01]$. There was a significant main effect of cue $[F(1,9)=6.6, p=$ $.03]$. There was an interaction between cue and target dimension $[F(1,9)=5.87, p<.05]$. (See Table 2.)

\section{Discussion}

The results show that it was possible to modulate the eye movements by advance cuing in a saccadic localization task. If the cue was identical to the target, the observers were faster to make an eye movement than if it was neutral. So, even though the saccadic latencies were around $190 \mathrm{msec}$ in Experiment 3, similar to those obtained in Experiment 2, there was room for improvement in the present experiment. This finding suggests that it is unlikely that the absence of top-down modulation in a saccadic localization task in Experiment 2-in which the dimension was cued by a word cue - was possibly due to a ceiling effect.

This result is in line with the results of Theeuwes et al. (2006, Experiments 4 and 5), which showed a validity effect of a picture cue in a compound search task, even if the cue was not predictive. Furthermore, it is in agreement with several studies showing intertrial priming effects (Found \& Müller, 1996; Hillstrom, 2000; Maljkovic \& Nakayama, 1994; McPeek et al., 1999; Mortier et al., 2005;

Table 2

Experiment 3: Mean Reaction Time (in Milliseconds) for Target-Present Trials As a Function of the Picture Cue (Valid or Neutral) and Target Dimension (Color or Orientation)

\begin{tabular}{clc}
\hline & \multicolumn{2}{c}{ Picture Cue } \\
\cline { 2 - 3 } Target Dimension & Valid & Neutral \\
\hline Color & $177^{*}$ & $191^{*}$ \\
Orientation & 192 & 202 \\
\hline
\end{tabular}

${ }^{*} p \leq .05$
Müller et al., 1995; Olivers \& Humphreys, 2003; Theeuwes et al., 2006). In these studies, responses to a given target were speeded up if the previous trial contained the same target. Intertrial facilitation is generally explained by perceptual processes. For example, in McPeek et al., observers had to make a speeded saccade to a pop-out color target. The saccadic latencies decreased when the target color was repeated. McPeek et al. reasoned that this priming of focal attention by target repetition facilitated the saccades. Taking together the results of previous studies, the effect on saccadic latencies induced by a picture cue seems to be the result of bottom-up priming of the allocation of attention to the target, which resulted in a faster initiation of a saccade.

The results of Experiments 1A-2 suggest that top-down information about the dimension of the upcoming target facilitates detection responses, regardless of the mode of responding (manual or saccadic), but not the speed of the saccadic latencies with which a target can be localized. ${ }^{1}$ The results of Experiment 3 showed that the absence of the cuing effect in Experiment 2 was not due to a ceiling effect. If localization responses cannot be modulated by top-down information, then changing the mode of responding to provide longer RTs should not make a difference. In Experiment 4, we combined a manual response with a localization task.

\section{EXPERIMENT 4}

Instead of using a saccadic response, the participants manually indicated where the target was located. If the absence of the cuing effect in the localization task of Experiment 2 was caused by the type of response (and the possible ceiling effect associated with it), one would expect that a cuing effect would be observed when the response is changed to manual. On the other hand, if top-down information cannot speed up localization, regardless of the response mode (manual or saccadic), one would predict no cuing effect in a localization task with manual responses.

\section{Method}

The participants had to locate the targets with a manual response. The participants pressed the " $z$ " key when the target was presented on the left side of the fixation point and pressed the "/" key when the target was presented on the right side of the fixation point. The participants were instructed not to move their eyes during the trials. Trials in which an eye movement of $3^{\circ}$ or more was made were removed from the data analysis. Between the trials, the participants were allowed to move their eyes.

\section{Results}

RTs of more than $1,000 \mathrm{msec}$ were counted as errors $(0.07 \%)$ and were excluded from the analysis. Also, trials in which an eye movement was made $(3.93 \%)$ were excluded from the analysis. The incorrect responses (5.54\%) were submitted to an ANOVA, with the factors cue (valid or neutral) and target dimension (orientation or color). There were no significant effects (all $F_{\mathrm{s}}<1$ ).

A within-subjects ANOVA was performed on the manual response latencies with cue (valid or neutral) and tar- 
get dimension (color or orientation) as factors. No effect of cue was found $(F<1)$, no effect of target dimension was found $(F<1)$, and no interaction was found between cue type and target dimension $(F<1)$. Unlike in the previous experiments, we failed to find a main effect of target dimension.

\section{Discussion}

The results of Experiment 4 show that advance information of the dimension of the target does not in any way affect localization of the cued target. Note that the manual RTs in this experiment, in which no cuing effect was found, were around $410 \mathrm{msec}$, which is comparable to those obtained in Experiment 1A, in which we found the classic cuing effect. This suggests that the absolute speed of responding is not a determining factor in obtaining cuing effects. ${ }^{2}$

\section{GENERAL DISCUSSION}

The present study adds to the ongoing debate concerning the possibility of top-down control in selection. In the dimensional weighting account (Müller et al., 2003) and in guided search (e.g., Wolfe et al., 2003), it is assumed that participants can use cues to allocate attentional weight to the likely target dimension. In other words, it is assumed that the salience map can be altered by top-down information in advance of target selection (Wolfe, 1994;
Wolfe et al., 2003; Wolfe et al., 2004; Müller et al., 1995; Müller et al., 2003). Most evidence for this comes from experiments showing that top-down information speeds up detection responses with singleton targets (Müller et al., 1995; Müller et al., 2003). In contrast, others have argued that the salience map cannot be modulated by topdown information in advance of target selection. They have made their claims on the basis of the absence of topdown guidance effects in compound search tasks (e.g., Cohen \& Magen, 1999; Mortier et al., 2005; Theeuwes et al., 2006; Theeuwes \& Van der Burg, 2007). However, directly comparing performance in the two tasks may be flawed because compound search tasks are more difficult and have slower RTs than do singleton detection tasks (Müller \& Krummenacher, 2006). The present study was designed so that we could investigate whether top-down modulation is possible in a task (target localization) with a level of task difficulty and range of RTs similar to those of a singleton detection task. We replicated findings that singleton detection is speeded by top-down knowledge of target dimension but found no effects of such knowledge on localization performance. ${ }^{3}$

One might argue that the trial-by-trial cuing procedure used here is simply not effective enough to modulate saccade-to-target latencies. However, if the cue was identical to the upcoming target, saccade-to-target latencies were decreased relative to when the cue was neutral (Experiment 3), probably as a result of bottom-up priming. This

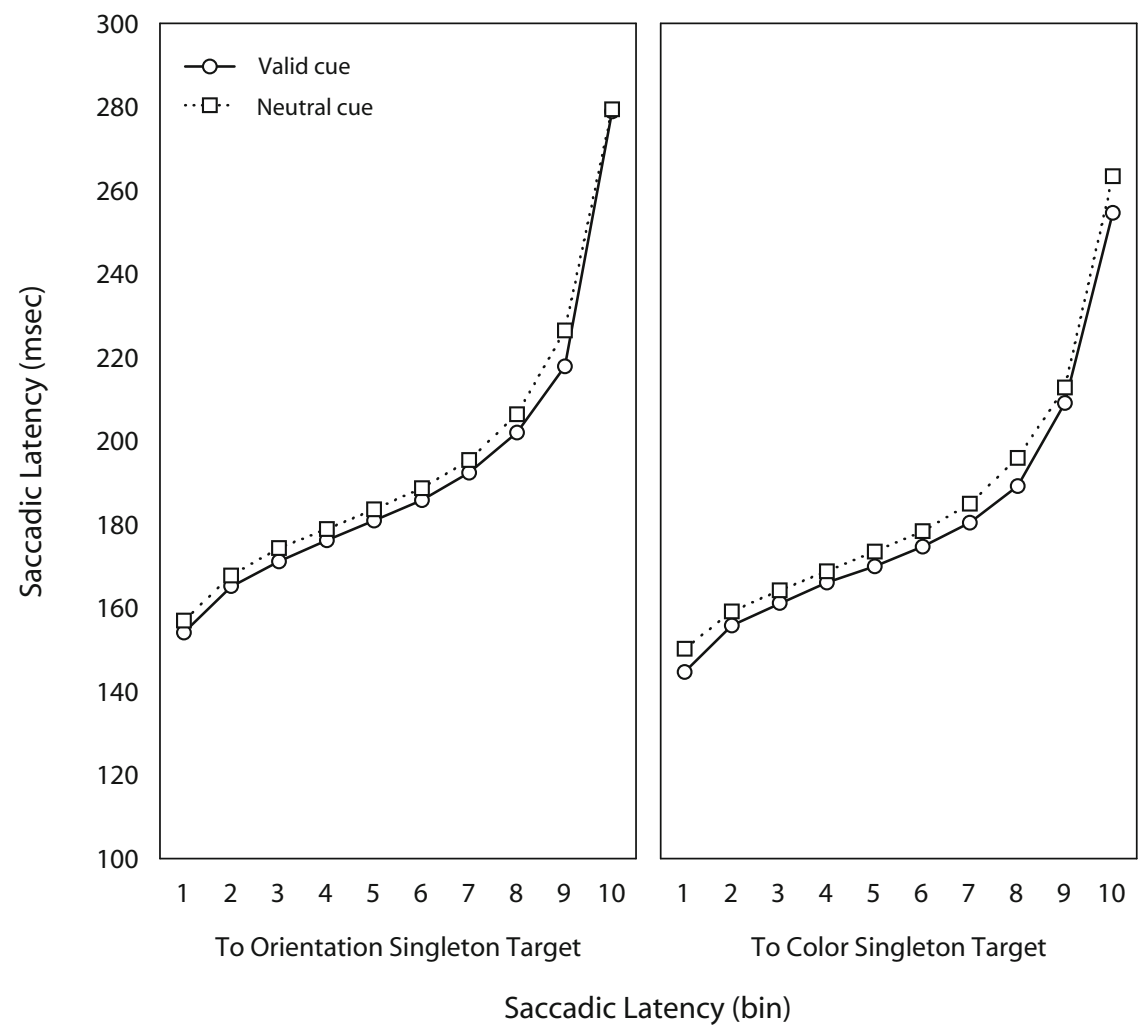

Figure 3. Saccadic latencies as a function of saccade percentiles (1-10 bins), cue validity, and target dimension in Experiment 2. 
finding shows that it is possible to facilitate the saccadic latencies with information provided by advance cuing.

Our results are inconsistent with the view that top-down knowledge of target dimensions speeds up selection of the target by increasing its salience. If this were the case, target localization should also have been affected by topdown knowledge. ${ }^{4}$ The present results suggest that topdown knowledge of target identity affects performance after the singleton target has been selected, probably at the level of response selection (Cohen \& Magen, 1999; Mortier et al., 2005; Theeuwes et al., 2006). The question then arises why such top-down knowledge would affect response selection in some tasks (i.e., detection) and not in others (i.e., localization and compound search).

First, why do word cues actually aid detection performance? Several authors have suggested that singleton detection is a signal detection task (e.g., Olivers \& Meeter, 2006; Palmer, 1995). In a signal detection task, participants have to decide whether there is a deviating element in the display. In order to prevent mistaking visual noise for a deviating element, the participants set a criterion that allows them to discount the visual noise but to identify targets as such. In search tasks in which the target can pop out on different visual dimensions, evidence from each of these dimensions is relevant for the response. Top-down knowledge of the target dimension may aid the participants, in that they can discount evidence from uncued dimensions. If the target is, for example, either a green or an oblique line, an advance cue that a color target is coming might lead the observer to discount evidence from the orientation dimension. In turn, the observer may discount visual noise from that dimension and may therefore be able to reach the detection criterion faster than was possible without the benefit of an advance cue.

These ideas are similar to those of Cohen and Shoup (1997), who suggested that responses to visual stimuli are made in dimension-specific response modules. When making a detection response, for example, the response that a target is present could be made on the basis of information from the color dimension or from the orientation dimension but not from both. Krummenacher et al. (2001, 2002) provided strong evidence against this possibility, by showing that responses to redundant targets, which pop out on two or more dimensions, were faster than could be explained by independent consideration of different visual dimensions. Instead of arguing for a complete independence of dimension-specific response modules (Cohen \& Shoup, 1997), we suggest that targetrelevant signals are derived from each visual dimension separately. Then these signals are added up and compared with a response threshold to generate a detection response. Accordingly, word cuing the dimension affects the degree to which different dimensions contribute to this response (see Figure 4).

Why would such reasoning not apply to localization or compound tasks? With regard to localization tasks, there are at least two possibilities. First, localization, at least in fast singleton search tasks, could rely on a master saliency map in which all visual dimensions are integrated
(Li, 2002). This map is, at least initially, impenetrable for top-down knowledge (see Donk \& van Zoest, 2008) and cannot be used to generate a detection response: The saliency map can guide attention but cannot be read to determine whether a threshold is reached to detect a target. This is a particularly appealing account of localization by eye movements, since observers in so-called oculomotor capture paradigms are often unaware of eye movements to salient distractors (Kramer, Hahn, Irwin, \& Theeuwes, 2000). Manual localization responses may be based on a shift of spatial attention that is driven by such a saliency map and that is thus also immune to top-down biases. The account is also consistent with the view that there are two main visual processing pathways (Milner \& Goodale, 1995; Ungerleider \& Mishkin, 1982): the ventral and the dorsal pathway. Although each pathway probably supports detection and localization to some extent, they use visual information in different ways. The ventral pathway is specialized for object identification (what), whereas the dorsal pathway is specialized for localization of objects (where, how). It is plausible that only the ventral pathway benefits from top-down information about the characteristics of an object, since this pathway is mainly dedicated to object perception and identification.

A second and alternative account is that localization responses are based on the same (or similar) noisy signals as are detection tasks but that these signals play a different role in the decision to be made. In detection tasks, signals have to be compared with a fixed threshold and implicitly with visual noise. In localization tasks, it is the location of the maximum signal that is relevant for the response. This means that signals from different locations have to be compared with one another and not with visual noise. If visual noise across the visual field is partly correlated (i.e., if it is a function of time more than of space), it is expected not to play a large role in the comparison of different locations. Limiting visual noise by de-emphasizing one visual dimension over the others would then lose its usefulness.

Both accounts could also explain findings from compound search tasks. Such tasks are generally thought to have two steps: localization of the target and a response based on some characteristic other than the characteristic that defines the target as a target. If localization of the target is not influenced by knowledge of the target-defining feature, neither should compound tasks.

It is possible to construct a variant of the ideas presented above that would come down to a dimension weighting account of response selection. As was discussed in the introduction, Müller et al. (2003; see also Wolfe et al., 2003; Wolfe et al., 2004) suggested that top-down knowledge can alter the attentional weights with which dimensional or feature maps contribute to an overall salience map. In turn, the salience map determines what is selected. We suggest that observers can alter the weights with which dimensions contribute to response selection processes. A localization response can be based directly on the information in the salience map, whereas in a detection response, the information of the salience map must be compared with a fixed criterion. 


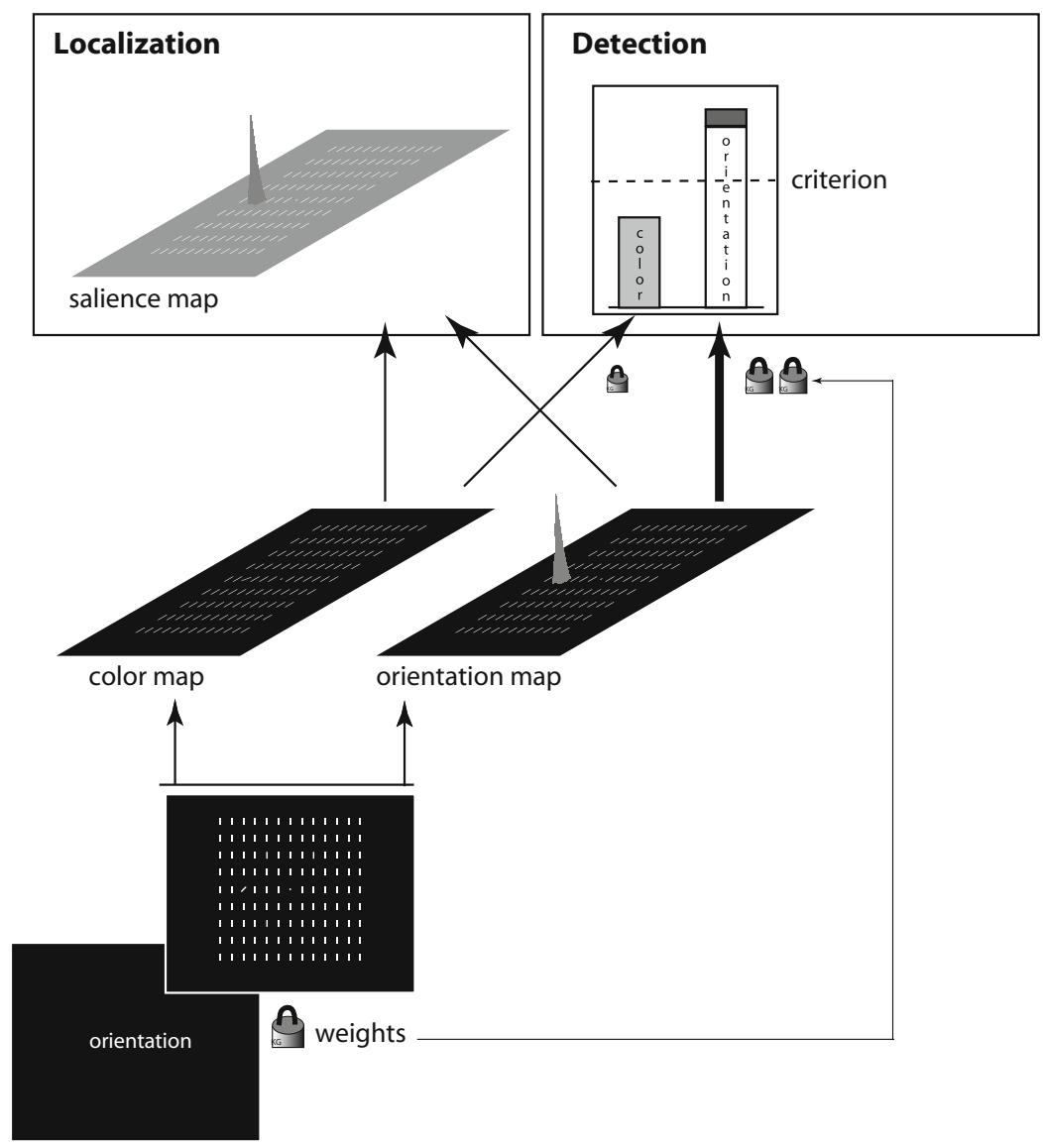

Figure 4. Possible explanation of our main findings. Signals from the stimulus display, here containing an orientation target, were analyzed on dimension-specific maps (shown: a color map and an orientation map). In these maps, stimuli that differed from their neighbors generated stronger signals than did items that resembled their neighbors. The resulting signals were integrated into a saliency map and into signals that were used to decide the presence or absence of a stimulus. To avoid false positives, detection signals were weighted by dimension weights. If the observer knew that a target, if present, would have a unique orientation (shown: an orientation cue), he or she could increase the weight of the orientation dimension, and so avoid being misled by visual noise on the color map. Localization responses were made to the maximum value on the saliency map and were thus not based on weighted signals. Visual noise was present in the picture as two larger signals on the color map. We assume that visual noise varied more strongly from trial to trial than between stimuli presented at the same moment.

Although the two accounts seem very similar, they make different predictions. The attentional weight account predicts that top-down knowledge of target features should affect all visual search tasks. Our account predicts that such knowledge affects only those tasks in which evidence from different visual dimensions is used in response selection. Concretely, top-down cuing the target dimension (i.e., with a word cue) should affect detection tasks but not compound search or target localization tasks. This is indeed what the present results show, as do those of Theeuwes et al. (2006) and those of Mortier et al. (2005). Furthermore, our account is in line with the recent findings of Chan and Hayward (2009). They found a crossdimensional cost in a detection task but not in a localization task or in a compound task.
Moreover, our account - but not the attentional weighting account - predicts that top-down knowledge in detection tasks also speeds responses on target-absent trials (because in target-absent trials, top-down knowledge can aid selection of the response but not attentional selection, because there is no target to select). This has indeed been reported (Meeter \& Theeuwes, 2006).

A counterexample would seem to be the recent results of Müller and Krummenacher (2006). They found an 11 -msec cuing effect in a compound search task. A difference between this study and previous ones is that after each trial, participants had to rate whether they had found the cue useful. Müller and Krummenacher argued that this made the participants pay attention to the cue, whereas in other experiments, the cue was ignored. We would sug- 
gest that it made the task a dual task, in which the participants paid attention to their performance. It could be that a cuing effect was obtained because of this extra task (rating task). However, how this extra task affected performance in the compound search task is not clear. Therefore, it is difficult to draw conclusions from a dual task in order to explain performance in a single task. Admittedly, this is a somewhat ad hoc explanation, and further studies will be needed to establish that cuing target dimensions truly does not affect compound search tasks.

In summary, we replicated findings that top-down knowledge of the target dimension can facilitate singleton detection. No top-down effects were found, however, on localization tasks. This pattern of results was found independent of whether saccadic or manual responses were made. We suggest that this is the case because top-down knowledge of the target dimension speeds response selection in singleton detection tasks in which different dimensions must be integrated to come to a response but not in other singleton search tasks.

\section{AUTHOR NOTE}

This research was supported by Dutch Scientific Research (NWO) Grant 42520204 to J.T. and a VENI grant from the same organization to M.M. Correspondence concerning this article should be addressed to K. Mortier, Department of Psychology, Vanderbilt University, Nashville, TN 37203 (e-mail: karen.mortier@vanderbilt.edu).

\section{REFERENCES}

Bichot, N. P., \& Schall, J. D. (2002). Priming in macaque frontal cortex during popout visual search: Feature-based facilitation and location-based inhibition of return. Journal of Neuroscience, 22, 4675-4685.

Chan, L. K. H., \& Hayward, W. G. (2009). Feature integration theory revisited: Dissociating feature detection and attentional guidance in visual search. Journal of Experimental Psychology: Human Perception \& Performance, 35, 119-132. doi:10.1037/0096-1523.35.1.119

Cohen, A., \& MAgen, H. (1999). Intra- and cross-dimensional visual search for single-feature targets. Perception \& Psychophysics, 61, 291-307.

Cohen, A., \& Shoup, R. (1997). Perceptual dimensional constraints in response selection processes. Cognitive Psychology, 32, 128-181. doi:10.1006/cogp.1997.0648

Corbetta, M., \& Shulman, G. L. (2002). Control of goal-directed and stimulus-driven attention in the brain. Nature Reviews Neuroscience, 3, 201-215. doi:10.1038/nrn755

Donk, M., \& van Zoest, W. (2008). Effects of salience are shortlived. Psychological Science, 19, 733-739. doi:10.1111/j.1467-9280 .2008.02149.x

Duncan, J. (1985). Visual search and visual attention. In M. I. Posner \& O. S. M. Marin (Eds.), Attention and performance XI (pp. 85-106). Hillsdale, NJ: Erlbaum.

Egeth, H. (1977). Attention and preattention. In G. H. Bower (Ed.), The psychology of learning and motivation (Vol. 11, pp. 277-320). New York: Academic Press.

Found, A., \& MülleR, H. J. (1996). Searching for unknown feature targets on more than one dimension: Investigating a "dimensionweighting" account. Perception \& Psychophysics, 58, 88-101.

Hillstrom, A. P. (2000). Repetition effects in visual search. Perception \& Psychophysics, 62, 800-817.

Kramer, A. F., Hahn, S., Irwin, D. E., \& Theeuwes, J. (2000). Age differences in the control of looking behavior: Do you know where your eyes have been? Psychological Science, 11, 210-217.

Krummenacher, J., Müller, H. J., \& Heller, D. (2001). Visual search for dimensionally redundant pop-out targets: Evidence for parallel-coactive processing of dimensions. Perception \& Psychophysics, 63, 907-917.

Krummenacher, J., Müller, H. J., \& Heller, D. (2002). Visual search for dimensionally redundant pop-out targets: Redundancy gains in compound tasks. Visual Cognition, 9, 801-837. doi:10.1080/ 13506280143000269

Kumada, T. (2001). Feature-based control of attention: Evidence for two forms of dimension weighting. Perception \& Psychophysics, 63, 698-708.

LI, Z. (2002). A saliency map in primary visual cortex. Trends in Cognitive Sciences, 6, 9-16. doi:10.1016/S1364-6613(00)01817-9

MaljKovic, V., \& NakaYama, K. (1994). Priming of pop-out: I. Role of features. Memory \& Cognition, 22, 657-672.

McPeek, R. M., Maljkovic, V., \& Nakayama, K. (1999). Saccades require focal attention and are facilitated by a short-term memory system. Vision Research, 39, 1555-1566. doi:10.1016/S0042 -6989(98)00228-4

Meeter, M., \& Theeuwes, J. (2006). Cuing the dimension of a distractor: Verbal cues of target identity also benefit same-dimension distractor singletons. Psychonomic Bulletin \& Review, 13, 118-124.

Milner, A. D., \& Goodale, M. A. (1995). The visual brain in action. Oxford: Oxford University Press.

Mortier, K., Theeuwes, J., \& Starreveld, P. A. (2005). Response selection modulates visual search within and across dimensions. Journal of Experimental Psychology: Human Perception \& Performance, 31, 542-557. doi:10.1037/0096-1523.31.3.542

Müller, H. J., Heller, D., \& Ziegler, J. (1995). Visual search for singleton feature targets within and across dimensions. Perception \& Psychophysics, 57, 1-17.

Müller, H. J., \& Krummenacher, J. (2006). Locus of dimension weighting: Preattentive or postselective? Visual Cognition, 14, 490513. doi:10.1080/13506280500194154

Müller, H. J., Reimann, B., \& Krummenacher, J. (2003). Visual search for singleton feature targets across dimensions: Stimulus- and expectancy-driven effects in dimensional weighting. Journal of Experimental Psychology: Human Perception \& Performance, 29, 10211035. doi:10.1037/0096-1523.29.5.1021

Olivers, C. N. L., \& Humphreys, G. W. (2003). Attentional guidance by salient feature singleton depends on intertrial contingencies. Journal of Experimental Psychology: Human Perception \& Performance, 29, 650-657. doi:10.1037/0096-1523.29.3.650

Olivers, C. N. L., \& Meeter, M. (2006). On the dissociation between compound and present/absent tasks in visual search: Intertrial priming is ambiguity-driven. Visual Cognition, 13, 1-28. doi:10.1080/13506280500308101

PALMER, J. (1995). Attention in visual search: Distinguishing four causes of a set-size effect. Current Directions in Psychological Science, 4, 118-123. doi:10.1111/j.1467-8721.1995.tb00258.x

Rauschenberger, R. (2003). Attentional capture by auto- and allocues. Psychonomic Bulletin \& Review, 10, 814-842.

Theeuwes, J. (1992). Perceptual selectivity for color and form. Perception \& Psychophysics, 51, 599-606.

Theeuwes, J., \& Godisn, R. (2001). Attention and oculomotor capture. In C. Folk \& B. Gibson (Eds.), Attraction, distraction, and action: Multiple perspectives on attentional capture (pp. 121-150). Amsterdam: Elsevier.

Theeuwes, J., Kramer, A. F., \& Atchley, P. (1999). Attentional effects on preattentive vision: Spatial precues affect the detection of simple features. Journal of Experimental Psychology: Human Perception \& Performance, 25, 341-347.

Theeuwes, J., Kramer, A. F., Hahn, S., Irwin, D. E., \& Zelinsky, G. J. (1999). Influence of attentional capture on oculomotor control. Journal of Experimental Psychology: Human Perception \& Performance, 25, 1595-1608. doi:10.1037/0096-1523.25.6.1595

Theeuwes, J., ReimanN, B., \& Mortier, K. (2006). Visual search for featural singletons: No top-down modulation, only bottom-up priming. Visual Cognition, 14, 466-489. doi:10.1080/13506280500195110

Theeuwes, J., \& VAN Der Burg, E. (2007). The role of spatial and nonspatial information in visual selection. Journal of Experimental Psychology: Human Perception \& Performance, 33, 1335-1351. doi:10.1037/0096-1523.33.6.1335 
Treisman, A. M. (1988). Features and objects: The fourteenth Bartlett memorial lecture. Quarterly Journal of Experimental Psychology, 40A, 201-237. doi:10.1080/02724988843000104

UngerLeIDER, L. G., \& MishKin, M. (1982). Two cortical visual systems. In D. J. Ingle, M. A. Goodale, \& R. J. W. Mansfield (Eds.), Analysis of visual behavior (pp. 549-586). Cambridge, MA: MIT Press.

van Zoest, W., \& Donk, M. (2005). The effects of salience on saccadic target selection. Visual Cognition, 12, 353-375. doi:10.1111/j.1467 $-9280.2008 .02149 . \mathrm{x}$

van Zoest, W., Donk, M., \& Theeuwes, J. (2004). The role of stimulusdriven and goal-driven control in saccadic visual selection. Journal of Experimental Psychology: Human Perception \& Performance, 30, 746-759. doi: 10.1037/0096-1523.30.4.746

Wolfe, J. M. (1994). Guided Search 2.0: A revised model of visual search. Psychonomic Bulletin \& Review, 1, 202-238.

Wolfe, J. M., Butcher, S. J., Lee, C., \& Hyle, M. (2003). Changing your mind: On the contributions of top-down and bottom-up guidance in visual search for feature singletons. Journal of Experimental Psychology: Human Perception \& Performance, 29, 483-502. doi:10.1037/0096-1523.29.2.483

Wolfe, J. M., Horowitz, T. S., Kenner, N., Hyle, M., \& Vasan, N. (2004). How fast can you change your mind? The speed of topdown guidance in visual search. Vision Research, 44, 1411-1426. doi:10.1016/j.visres.2003.11.024

\section{NOTES}

1. The percentage of eye movements captured by the target singleton in Experiment 1B was very low. This finding seems in conflict with previous findings reporting on average $30 \%$ oculomotor capture in similar paradigms (Theeuwes, Kramer, Hahn, Irwin, \& Zelinsky, 1999; van Zoest \& Donk, 2005). However, in our study, saccadic latencies were relatively high. Evidence suggests that long-latency saccades are much more goal-driven (van Zoest, Donk, \& Theeuwes, 2004), hence explaining the absence of oculomotor capture in the present experiment.

2 . Another difference between detection and localization tasks is that a target is present on $50 \%$ of the trials in the former, but on $100 \%$ of the trials in the latter. We tested whether this explains the divergent results between detection and localization tasks by giving 8 participants a manual localization task in which the target was present on only $50 \%$ of trials. On the trials in which no target was present, no response had to be given. Performance was not faster when the target dimension was cued $(M=436 \mathrm{msec})$ than when a neutral cue was given $(M=432 \mathrm{msec})$ $[F(1,7)<1]$

3. We compared Experiments 2 and 4 with Experiments 1A and 1B with cue and target dimension as within-subjects factors and experiment as a between-subjects factor. The planned comparison of localization task experiments ( 2 and 4 ) with detection task experiments (1A and 1B), collapsed over the two different target dimensions, showed a significant interaction between cuing and the type of task $[F(1,38)=11.76, p<$ $.01]$. This between-experiments analysis shows that the null effects in the localization tasks are indeed different from the cuing effects obtained in the detection tasks.

Furthermore, we divided the individual mean saccadic latencies for Experiment 2 in percentiles (bins) from the fastest to the slowest, separately for the valid cue trials and the neutral cue trials. A three-way ANOVA was performed with bin, cue, and target dimension as withinsubjects factors. Besides a main effect of bin, there was a main effect of target dimension $[F(1,9)=49.1, p<.001]$ and an interaction between bin and target dimension $[F(9,81)=3.15, p<.01]$, but more important, bin did not interact with the cuing effect $(F<1)$. This result indicates that even the slower saccadic latencies are not affected by the cue. (See Figure 3.)

4. One could argue that perhaps knowledge of features instead of knowledge of dimensions will affect target localization. This is, however, highly unlikely, because in the present series of experiments, only one feature for each dimension (color, green; orientation, right tilted) was presented, enabling the observers to select on the basis of single features. Indeed, Theeuwes et al. (2006, Experiments 2, 3, and 4) showed that irrespective of whether the dimension or the exact feature of the upcoming target singleton was cued, the sensitivity in detecting a target singleton was not enhanced. Thus, even though feature-based modulation was possible in the present experiments, no effects were found on the localization task. Therefore, we do not think that feature-based top-down information would result in top-down modulation of target localization.

(Manuscript received March 25, 2009; revision accepted for publication July 27, 2009.) 